\title{
DNA binding of CPF1 is required for optimal centromere function but not for maintaining methionine prototrophy in yeast
}

\author{
J.Mellor, J.Rathjen, W.Jiang ${ }^{1,+}$ and S.J.Dowell \\ Department of Biochemistry, South Parks Road, Oxford, OX1 3QU, UK and ${ }^{1}$ Institute of Microbiology \\ and Molecular Biology, Frankfurter-Strasse 107, D-6300 Giessen, FRG
}

Received February 21, 1991; Revised and Accepted May 9, 1991

\begin{abstract}
The centromere and promoter factor 1 (CPF1) binds specifically in vitro and in vivo to an octanucleotide (RTCACRTG). This sequence is found in the centromere DNA element I (CDEI) of yeast centromeres and upstream from a number of transcription units including MET25, GAL2 and TRP1. Inactivation of the CPF1 gene results in three phenotypes; slow growth, a partial loss of centromere function and methionine auxotrophy. These phenotypes correlate well with the known binding sites for CPF1 and have led to the suggestion that CPF1 functions as a kinetochore protein at centromeres and as a transcriptional activator at promoters such as MET25. By analysing transcription from the MET25, GAL2, and TRP1 genes in cpf1 strains, we demonstrate that CPF1 plays no direct role in their transcriptional regulation. Further evidence in support of this comes from the analysis of point mutations in the basic region of CPF1 that affect DNA binding. A strain expressing a non-DNA bound form of CPF1 is phenotypically Met ${ }^{+}$, shows normal growth rate but has sub-optimal centromere function. We conclude that a DNA-bound form of CPF1 is required for the kinetochore function but not for maintaining methionine prototrophy.
\end{abstract}

\section{INTRODUCTION}

Centromeres of the yeast Saccharomyces cerevisiae share three highly conserved DNA sequence elements, designated CDEI, CDEII and CDEIII (1). The three element are found in a 120bp core sequence which is sufficient for complete centromere activity in vivo (2). CDEIII is the most important of these elements for centromere function as point mutations within CDEIII inactivate the centromere $(2,3)$. Point mutations at any position within the CDEI octanucleotide (RTCACRTG; $\mathbf{R}=$ purine) impair but do not abolish function (2). Chromosomes or centromere based plasmids carrying CDEI mutations are lost during mitotic growth at rates 2 to 60 fold higher than chromosomes or plasmids with wild type centromeres $(2,4,5,6)$. The highly conserved nature of the CDEI and CDEIII sequences and their functional importance is reflected in the fact that they are sites of interaction for sequence specific DNA binding proteins $(5,7,8,9,10,11)$. A gene encoding a CDEI binding protein has been cloned $(12,13,14)$ and its product, the centromere and promoter factor 1 (CPF1), is known to bind to CDEI of yeast chromosomes in vivo (14). Sequences related to the CDEI octanucleotide are found in the promoter regions of several $S$. cerevisiae genes (15). Four genes in the pathway for S-adenosyl-methionine biosynthesis carry $\mathrm{CDEI}$ in their promoters and this sequence has been shown to be associated with a transcriptional upstream activation site in MET25 (16). The CDEI motif is found close to the RNA initiation sites in the downstream TRP1 promoter and upstream from the GAL4 binding sites in the GAL2 promoter $(15,17,18)$. By constructing yeast strains lacking the centromere and promoter factor 1 (by disruption of the CPF1 gene; 19) it is possible to detect changes in the chromatin structure or the accessibility of CDEI to dimethyl sulphate in centromeres and at several promoters containing CDEI such as MET25, TRP1 and GAL2 (14). These structural changes can be related to the presence or absence of the CPF1 protein in vivo (14). The implication of these data is that CPF1 is not only a component of a yeast kinetochore by virtue of interactions at CDEI in the centromere but may also play a role in transcription from yeast promoters, as first suggested by Bram and Kornberg (15).

Further evidence to support this idea comes from the observation that strains containing gene disruptions in the CPF1 locus have pleiotrophic phenotypes $(12,13,14)$. In addition to suboptimal chromosome segregation (consistent with a role for CPF1 as a kinetochore protein), $c p f 1$ strains show a defect in their growth rate and an auxotrophic requirement for methionine. It seems reasonable to suggest that the methionine auxotrophy of $c p f l$ strains may be caused by an inability to transcribe MET25 and related genes. Duplicate CDEI motifs are also found in the promoter regions of MET2, MET3 and SAM2, genes which are co-regulated with MET25 (16).

CPF1 is a member of a diverse family of proteins from yeast, Drosophila and mammals involved in growth control and differentiation which contain a distinctive motif, the helix-loop-

\footnotetext{
+ Present address: Department of Biological Sciences, University of California, Santa Barbara, CA 93106, USA
} 
helix (HLH) $(14,20,21,22,23)$. A number of these proteins are specific DNA binding proteins and DNA binding can be correlated with a short stretch of basic amino acids immediately preceding the HLH region $(24,25)$. In all cases where a specific binding site is known, the protein recognises related motifs of the form CANNTG which resembles CDEI $(12,24,25)$. These HLH proteins can be divided into groups by their ability to bind in vitro to $\mathrm{CDEI}$-like sequences as homodimers and/or heterodimers (23). It is known that CPF1 binds to CDEI as a homodimer (14). By analogy with other proteins in this family, it is possible that CPF1 can form homodimers with itself and heterodimers with other as yet unknown proteins, thus extending the range of functions attributable to CPF1.

In this paper, we use two approaches to investigate the multifunctional nature of CPF1. The first approach is to analyse the expression of the MET25, GAL2 and TRP1 genes in a cpf1 strain. In the second approach we attempt to separate kinetochore and transcription functions, as measured by centromere stability and methionine prototrophy respectively, by introducing point mutations into the CPF1 protein. Contrary to expectations, no significant difference in the amount or the regulation of transcription from the $M E T 25, T R P 1$ or $G A L 2$ promoters in $c p f 1$ or wild-type strains is seen. This suggests that CPF1 has no direct role in the transcription of these genes. However, analysis of strains expressing mutant versions of CPF1 allows us to separate the role of CPF1 as a kinetochore protein from its role in maintaining methionine prototrophy. We present evidence suggesting that DNA bound CPF1 is required for a kinetochore function but not for maintaining the methionine prototrophy.

\section{MATERIALS AND METHODS}

\section{Strains, media and growth conditions}

S.cerevisiae strains. DBY745 ( $\alpha$ leu2-3, leu2-112, ura3-52, ade1-100). YAG 90 is isogenic with DBY745 and carries a URA3 insertion in the MscI site of CPF1 (14). YAG214 and YAG216 are isogenic with DBY745 but carry point mutations in the $C P F 1$ locus. All four strains were transformed following the procedure of Hinnen et al. (26) to leucine prototrophy with the $C E N$ based plasmid pRS315 (27). Strains were grown at $25^{\circ} \mathrm{C}$ in YEPD (1\% yeast extract, $2 \%$ peptone and $2 \%$ glucose), YEPGal (1\% yeast extract, $2 \%$ peptone and $2 \%$ galactose) or in a defined minimal media (SC; $6.7 \%$ yeast nitrogen base w/o amino acids, $1 \%$ glucose and complete amino acid mixture lacking selective amino acids as required; 28). 5'Fluoro-orotic acid (5'FOA) selection followed lithium acetate transformation with $30 \mu \mathrm{g}$ of linear fragments containing the $3 \mathrm{~Kb}$ BamHI CPFl gene or mutant derivatives into YAG90 cells at a density of no more than $5 \times 10^{6} / \mathrm{ml}(29,30)$. Cells were replica plated from YEPD onto defined minimal plates containing $5^{\prime} \mathrm{FOA}$ at $750 \mu \mathrm{g} / \mathrm{ml}$ and uracil at $40 \mu \mathrm{g} / \mathrm{ml}$ after 12 to 16 hours growth at $30^{\circ} \mathrm{C}$.

\section{DNA analysis}

Genomic DNA was isolated from uracil auxotrophs using the method of Holm (31), digested with BamHI or TaqI, electrophoretically separated on $1 \%$ agarose gels and transferred to nylon filters as described by Southern (32). Filters were hybridised with a $3 \mathrm{~kb}$ BamHI fragment from CPF1 (14) or to end labelled oligonucleotides used for in vitro mutagenesis so that single base changes could be detected (33).

\section{RNA analysis}

RNA was isolated from yeast grown in defined minimal media according to the method of Dobson et al. (34). RNA was quantified using Northern blots (35) and hybridised using as probes: $X b a I / E c o R V$ fragment from MET25 (16); $3.4 \mathrm{~Kb}$ $B a m \mathrm{HI} / E c o \mathrm{RI}$ fragment from the actin gene (36); 1.8kb EcoRI

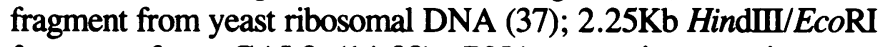
fragment from GAL2 $(14,38)$. RNA start site mapping was performed using primer extension analysis exactly as described by Rathjen and Mellor (39).

\section{DNA manipulations and plasmid constructions}

Restriction enzymes, T4 DNA ligase, T4 polynucleotide kinase, Klenow, Reverse transcriptase (MoMuLV) were obtained from BRL, Inc., Exonuclease III and Mung Bean Nuclease from Pharmacia and T7-GEN in vitro mutagenesis kit from USB. All enzymes and kits were used according to the manufacturers' protocols. A SphI/RsaI fragment from CPFl was cloned into M13 mp18 as a template for mutagenesis (14). Radioactively labelled isotopes were obtained from Amersham International. DNA sequencing was done using Sequenase V2.0 (USB). All other reagents were analytical grade.

The deletions in the RNA initiation region of the TRPI promoter were constructed as follows: A HincII $(-280$ in the TRP1 promoter)/PvuII (+300 in Interferon $\alpha$ cloned into a BamHI site at +1 ) was isolated from pMA1557-10 (40). This fragment was cut with MaeII and the two large fragments (HincII/MaeII (-41); MaeII (-31)/PvuII) isolated from a gel. The two fragments were incubated in a $10 \mu l$ sticky end ligation for three hours at room temperature, then HincII cut pSP46 added and a blunt end ligation reaction was made up to $20 \mu \mathrm{l}$. DNA from transformant containing a 90 base pair EcoRI $(-100) / \operatorname{BamHI}(-1)$ fragment was purified, the EcoRI/BamHI fragment isolated and cloned into EcoR1/BamHI cut pSP65. This creates a 10 base pair deletion between -41 and -31 and recreates a single MaeII site in the hybrid CACGTGA motif in a fragment called 13. To create further deletions around this site, pSP65-13 was cut with MaeII and the two large fragments isolated and either filled in with klenow or treated with exonuclease III (10 units/ $\mu \mathrm{g}$ of DNA; Pharmacia) for 1 to 5 minutes at $4^{\circ} \mathrm{C}$, then with 10 units of mung bean nuclease for 10 minutes at $37^{\circ} \mathrm{C}$. One reaction was set up with mung bean nuclease to remove the MaeII sticky ends. The resulting blunt ended fragments were ligated for 6 hours at room temperature in a $10 \mu$ reaction, cut with EcoRI and BamHI, then ligated with EcoRI/BamHI cut pSP65. DNA from transformants containing EcoRI/BamHI fragments smaller than 90 base pairs was purified, HincII/EcoRI fragments isolated, $3^{\prime}$ end labelled and sequenced using the Maxam and Gilbert procedure to confirm the integrity of the fragments and the extent of the deletions. pMA1557 formed the backbone of the molecules used to assess the effect of deletions in the TRPI promoter on mRNA initiation (40). To reconstruct the promoter, the HincII $(-280) / E c o \mathrm{RI}(-100)$ fragment from pMA1557-10 was cloned into HincII/EcoRI cut pSP46 and reisolated as a $B g l I I / E c o R I$ fragment. This was ligated with an equal amount of each $E c o \mathrm{RI}(-100) / \operatorname{BamHI}(-1)$ fragment and BamHI cut pMA1557 to yield a TRP1 promoter extending from -280 to -1 with modifications in the region around -30 . The promoter elements for transcription unit I are located between -395 and -293 and are thus deleted in these molecules (40). Results for only two derivatives of the TRPI promoter are shown in this paper. 


\section{Oligonucleotides}

Oligonucleotides were obtained from British Biotechnology Limited except where noted: For primer extension from human interferon- $\alpha$-2 sequences 5' GCCCACAGAGCAGCTTGAC 3' (Celltech) or TRP1 sequences 5' GGAATCTAGAGCACATTCTGC 3'. For site directed mutagenesis: H227 to N 5' GACTCCAATAAAGAAG 3'; E231 to A 5' GAAGTCGCAAGGCG 3'; RR234/5 to LL 5' GGCGTCTCCTCGAAAAC 3'. For DNA binding: E12 binding site 5' GATCTCAGGCAGCAGGTGTTGGGG $3^{\prime}$ and $5^{\prime}$ GATCCCCCAACACCTGCTGCCTGA 3' (a gift from Colin Goding); CPF1 binding site 23bp CDEI 5' GATCTGGCCACGTGACCGG 3' and 5' GATCCCGGTCACGTGGCCA 3'; Mutant CPF1 binding site C3 to $\mathrm{T}(\mathrm{m} 1) 5^{\prime}$ GATCTGGCTACGTGACCGG $3^{\prime}$ and $5^{\prime}$ GATCCCGGTCACGTAGCCA 3'; Mutant CPF1 binding site G8 to A (m2) 5' GATCTGGCCACGTAACCGG 3' and 5' GATCCCGGTTACGTGGCCA 3'.

In vitro transcription and translation.

The construction of pSP73-22 and the methods used to prepare protein in vitro have been described previously (14). pSP73-214, pSP73-216 and pSP73-217 were constructed by replacing a SphI/PstI fragment from pSP73-22 containing a modified polylinker with the equivalent fragment from the RF preps of M13 mp18 containing point mutations in the CPF1 fragment.

\section{Nuclear protein extracts}

Yeast nuclear extracts were prepared from strains grown in YEPD by spheroplasting with $0.2 \%$ glusulase (DuPont) in $1 \mathrm{M}$ sorbitol, washing spheroplasts twice in $1 \mathrm{M}$ sorbitol and lysing in one packed cell volume of $18 \%$ Ficoll 400 (Pharmacia), $20 \mathrm{mM}$ Hepes pH8.0 (BDH), $1 \mathrm{mM}$ EDTA using a glass rod at $4^{\circ} \mathrm{C} .20$ volumes of $9 \%$ Ficoll in the above buffer was added and the nuclei spun out for 30 minutes at $20 \mathrm{Kg}$, washed in 20 volumes of $1 \mathrm{M}$ sorbitol, spun at $9 \mathrm{Kg}$ for 10 minutes then resuspended in 1 packed cell volume of buffer $\mathrm{C}(0.42 \mathrm{M} \mathrm{NaCl}, 25 \%$ glycerol, $20 \mathrm{mM}$ Hepes pH8.0, 1mM EDTA, 1mM DTT, 1mM PMSF (Sigma)) and stirred on ice for 45 minutes. An equal volume of buffer $\mathrm{C}$ was added and the mixture spun at $50 \mathrm{Kg}$ for 30 minutes at $4^{\circ} \mathrm{C}$. The supernatant was dialysed overnight into $0.1 \mathrm{M} \mathrm{KCl}$, $20 \%$ glycerol, 20mM Hepes pH8.0, 1mMEDTA, 1mMDTT, $1 \mathrm{mM}$ PMSF and stored at $-70^{\circ} \mathrm{C}$. Protein estimation was performed as described by Bradford (1976). $15 \mu \mathrm{g}$ of extract were used in each binding reaction.

\section{Band shift analysis}

Band shift analyses were performed essentially as described by Jiang and Philippsen except that different ${ }^{32} \mathrm{P}$ labelled CDEI

Table I. Metabolites able to satisfy methionine auxotrophy of cpf1 strains

\begin{tabular}{lll}
\hline L-methionine & $1 \mathrm{mM}$ and $0.1 \mathrm{mM}$ & +++ \\
S-adenosyl-L-methionine & $0.2 \mathrm{mM}$ and $0.1 \mathrm{mM}$ & +++ \\
L-cysteine & $1 \mathrm{mM}$ and $0.2 \mathrm{mM}$ & +++ \\
DL-homocysteine & $2 \mathrm{mM}$ & +++ \\
& & \\
L-methionine & $0.01 \mathrm{mM}$ and $0.005 \mathrm{mM}$ & ++ \\
L-cysteine & $0.01 \mathrm{mM}$ & ++ \\
DL-homocysteine & $1 \mathrm{mM}$ & ++ \\
DL-homoserine & $2 \mathrm{mM}$ and $0.1 \mathrm{mM}$ & $+/-$ \\
DL-homocysteine & $0.1 \mathrm{mM}$ & $+/-$ \\
& & \\
L-homocystine & $1 \mathrm{mM}$ and $0.1 \mathrm{mM}$ & - \\
\hline
\end{tabular}

fragments or oligonucleotides were used (see figure legends; 10). Oligonucleotides were filled in to flush the ends with Klenow polymerase before use. $\left[\alpha^{-}{ }^{32} \mathrm{P}\right] \mathrm{dATP}(3000 \mathrm{Ci} / \mathrm{mmol})$ was included to radioactively label the DNA.

\section{Mitotic stability assay}

The stability assay was performed as described earlier $(3,14,41)$. The plasmid pRS315 has the LEU2 gene, CEN6 and ARSH4 and
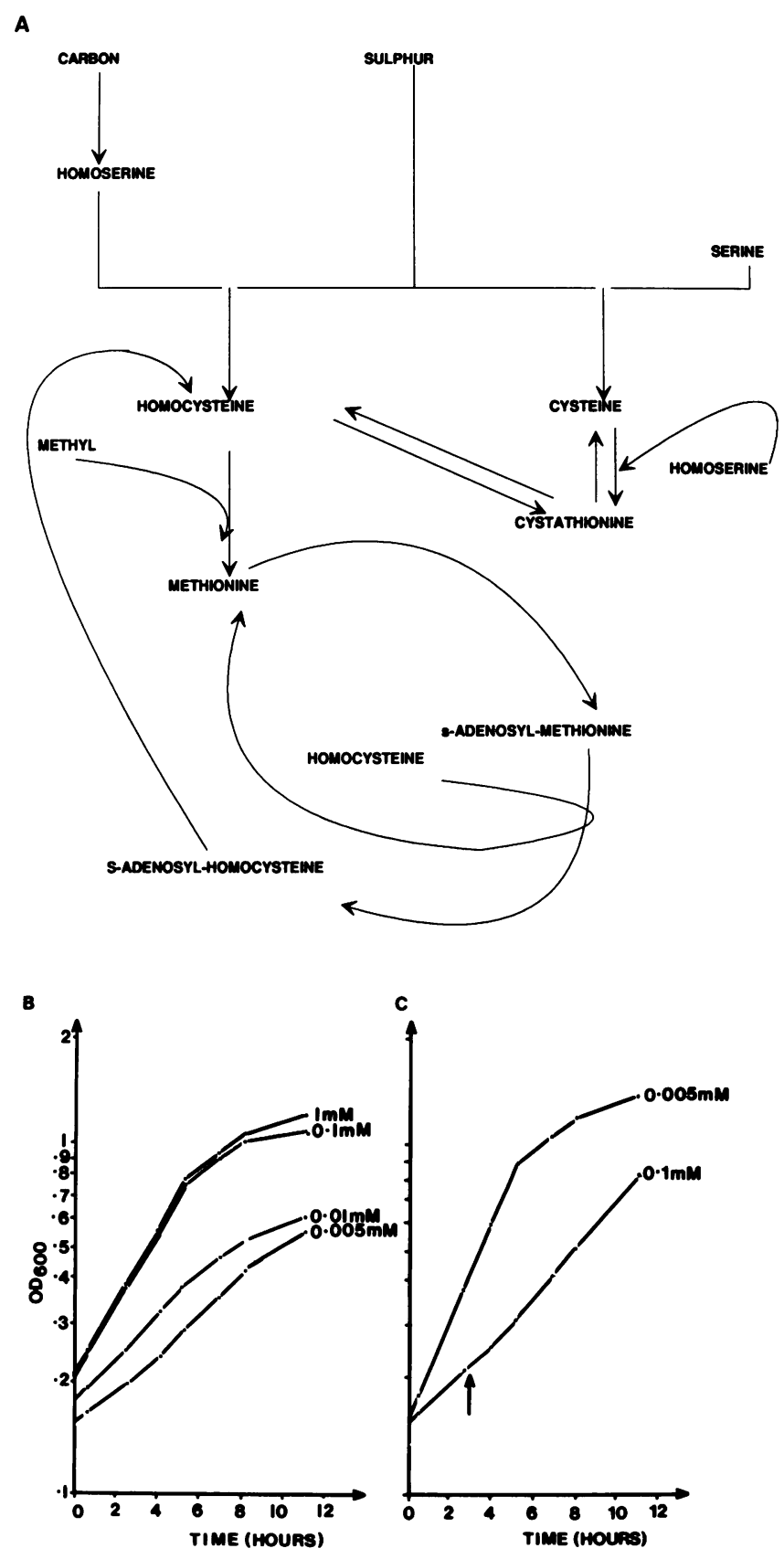

Figure 1.A. An outline of the methionine/S-adenosylmethionine biosynthetic pathway in yeast. B. Growth curves for yeast strain YAG90 (cpfl) in defined minimal medium supplemented with L-methionine at the concentrations given. C. Growth curve for DBY745 (CPF1) grown in defined minimal medium with $0.005 \mathrm{mM}$ methionine. Identical curves were seen for DBY745 grown at $0.01 \mathrm{mM}$, $0.1 \mathrm{mM}$ and $1 \mathrm{mM}$ methionine (not shown). Growth curve for YAG90 grown overnight in $0.01 \mathrm{mM}$ methionine and supplemented with $0.1 \mathrm{mM}$ methionine 2 hours $\mathbf{4 5}$ minutes after the first OD reading (marked by arrow). Growth was followed by measuring the optical density of the cultures at $600 \mathrm{~nm}$. 
was transformed into yeast and transformants colony purified on selective plates. Eight individual transformants were picked at random and grown overnight at $25^{\circ} \mathrm{C}$ in selective media. The percentage of $\mathrm{LEU}^{+}$cells present in cultures at time 0 and after 10 generations of growth in YEPD were determined by plating on full medium and after 24 hours of growth by replica plating onto selective medium. The number of cell doublings was calculated by counting the total cell number at the beginning and end of non-selective growth at $25^{\circ} \mathrm{C}$. The calculation of the average plasmid loss rate per cell and generation for each culture was done as follows: $\mathrm{Ln} / \mathrm{Lo}=(1-\mathrm{R})^{\mathrm{n}}$, were $\mathrm{Ln}=$ number of $L E U^{+}$colonies after $\mathrm{n}$ generation; Lo $=$ number of $L E U^{+}$ colonies after 0 generation; $\mathrm{R}=$ loss rate; $\mathrm{n}=$ number of generations.

\section{RESULTS}

\section{Methionine concentration affects the growth rate of $c p f 1$ strains}

In order to determine further the auxotrophic requirements of $c p f 1$ strains, yeast were plated out on medium lacking methionine and supplemented with various metabolites at various
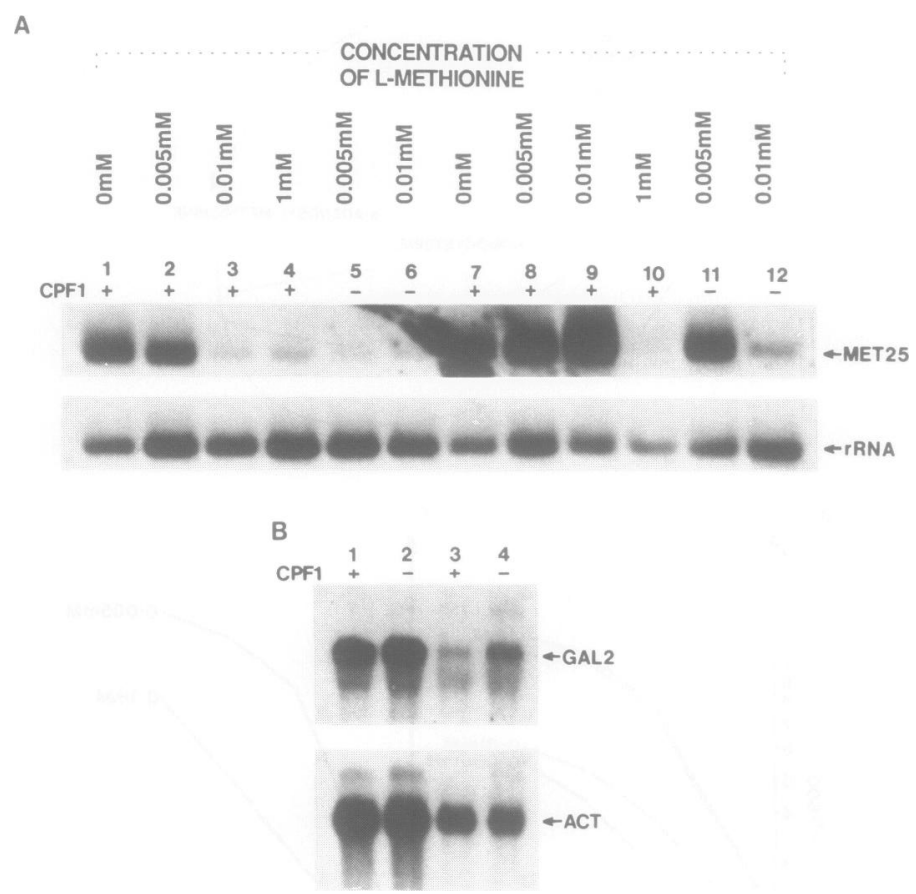

Figure 2. A. Northern blot probed with MET25 and ribosomal RNA (rRNA) specific probes. A wild type strain (DBY745; lanes 1 to 4 and 7 to 10)) and a cpfl strain (YAG90; lanes 5,6,11 and 12) were grown in $100 \mathrm{mls}$ of medium supplemented with various concentrations of L-methionine overnight to a cell density of $2-4 \times 10^{6}$ for DBY745 (lanes 1 to 4 ) and $1 \times 10^{6}$ for YAG90 (lanes 5 and 6). RNA was prepared from $50 \mathrm{ml}$ of the culture (lanes 1 to 6 ) and the same cultures were then left to grow for a further 9 hours to a cell density of approximately $1 \times 10^{7}$ for DBY745 (lanes 7 to 10 ) and approximately 4 to $5 \times 10^{6}$ for YAG90 (lanes 11 and 12) and RNA prepared from the remaining 50ml. The filter was probed with a MET25 specific probe, the probe washed off the filter and then rehybridised with a rRNA specific probe to control for the amount of RNA in each track of the gel. B. Northern blot of RNA prepared from cultures of DBY745 (CPF1; lanes 1 and 3) and YAG90 (cpfl; lanes 2 and 4) grown in YEPD (lanes 3 and 4 ) or YEPGal (lanes 1 and 2). The filter was hybridised to a $G A L 2$ specific probe, the probe washed off the filter and then rehybridised with an actin (ACT) specific probe. concentrations as outlined in Table 1 . The wild type (CPFI) strains such as DBY745 grow well on all media (data not shown). In addition to L-methionine, S-adenosyl-L-methionine, L-cysteine and DL-homocysteine were able to satisfy the auxotrophic requirements of YAG90 (cpfl). DL-homoserine had a marginal ability to sustain growth and no growth was seen on Lhomocystine. Sulphur containing compounds which are components of the lower part of the biosynthetic pathway are able to satisfy the methionine auxotrophy of cpfl strains (figure $1 \mathrm{~A} ; 42)$. These data did not allow us to draw any conclusions about possible defective steps in the methionine/S-adenosyl methionine biosynthetic pathway in $c p f l$ strains. However, we were surprised to find that the concentration of these metabolites, particularly L-methionine, S-adenosyl methionine and L-cysteine affects the growth rate of YAG90 but not of DBY745. At low concentrations $(0.01 \mathrm{mM}$ and $0.005 \mathrm{mM})$ of L-methionine, the growth rate of YAG90 in synthetic defined medium is 5 hours compared to 2.5 hours at higher concentrations $(0.1 \mathrm{mM}$ and $1 \mathrm{mM}$ )(figure 1B). If a culture grown overnight at a low concentration of L-methionine $(0.01 \mathrm{mM})$ is supplemented with a high $(0.1 \mathrm{mM})$ concentration a marked increase in growth rate

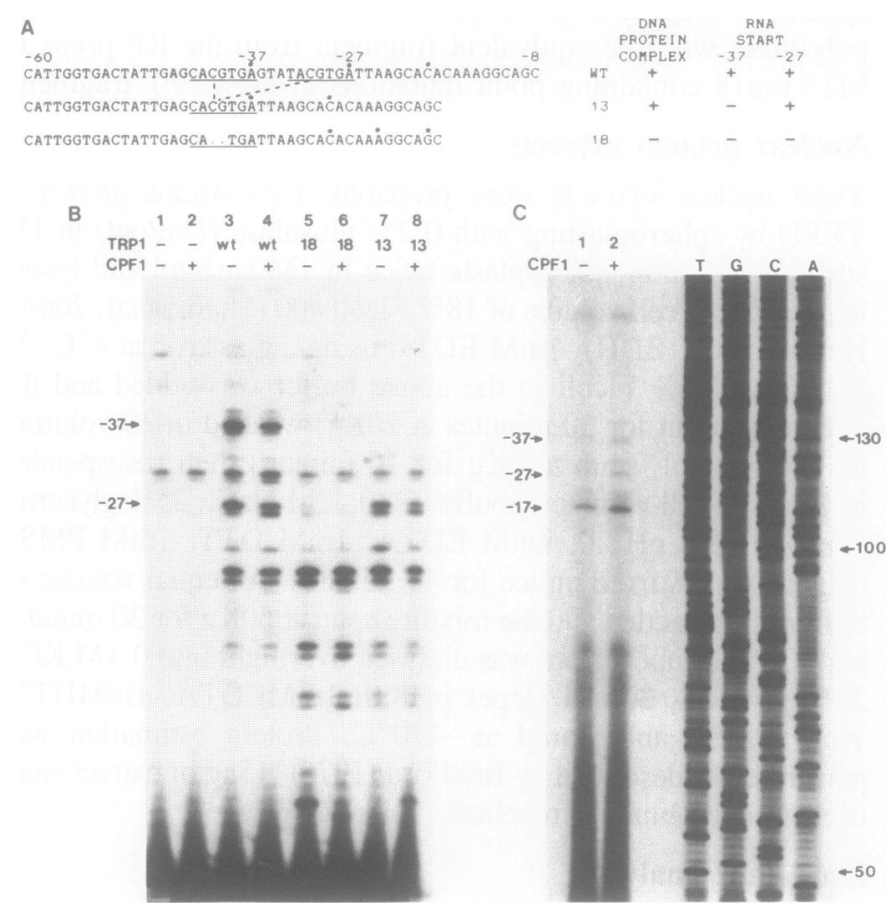

Figure $3 \mathrm{~A}$. Sequence of TRPI promoter fragments showing deletions around the CDEI motif. RNA start sites are indicated by dots over the initiating base. The ability of a fragment extending from -100 to -8 to form a DNA:protein complex with CPF1 as measured in a band shift assay and to initiate RNA at the -37 or -27 start sites on the TRPI promoter in pMA 1557 is indicated. B. RNA start site mapping on the TRPI promoter by primer extension. RNA prepared from strains YAG90 (lanes 1,3,5 and 7) and DBY745 (lanes 2,4,6 and 8) transformed with the promoter analysis vector pMA1557 (-; lanes 1 and 2), pMA1557 containing the wild type TRPI promoter fragment (wt; lanes 3 and 4 ), pMA1557 containing the TRPI promoter fragment with a deletion in the CACGTGA motif (18; 5 and 6) and pMA1557 containing the TRPI promoter fragment with one CACGTGA motif (13; lanes 7 and 8). An interferon specific primer was used. The positions of the -37 and -27 start sites are shown. C. Primer extension with a $T R P l$ specific primer $(+72$ to +92$)$ on RNA prepared from strains DBY745 (CPF1; lane 2) and YAG90 (cpfl; lane 1). Size markers indicating the length of the extension products are provided by a $\mathrm{M} 13 \mathrm{mp} 18$ sequence primed with a -40 primer. The position of the -27 and -37 extension products are indicated. 
is then apparent (figure 1C). In addition, strains carrying mutations in methionine biosynthetic genes, such as MET6, do not show a slow growth rate in the presence of low concentrations $(0.01 \mathrm{mM})$ of L-methionine (data not shown). One explanation for the effect of methionine concentration in $c p f 1$ strains might be that there is a defect in the uptake, sensing or localisation of methionine and other sulphur containing metabolites into cpfl cells. This is particularly pronounced when $c p f l$ strains are grown at low concentrations of these compounds.

\section{Transcription of genes containing CDEI is not regulated by CPF1}

MET25 transcription is inducible in cpf1 strains. The pattern of transcriptional regulation of methionine/S-adenosylmethionine biosynthetic genes such as $M E T 25$ has been established previously $(16,43)$. Expression is repressed at high concentrations of methionine and induced in the absence of or at low concentrations of methionine. RNA was prepared from cultures of DBY745 and YAG90 that had been grown in various concentrations of Lmethionine overnight. Cells were harvested at two cell densities. We had established previously concentrations of methionine suitable for growth of YAG90 strains and for induction of MET25 (See Table I). Note that once the MET25 RNA is induced in the cpfl strains, the methionine in the medium is exhausted and growth ceases at this point. Thus the cpfl cells were harvested at low cell density when the cultures are actively dividing and at a higher density when methionine in the medium was becoming exhausted. A representative experiment is shown in figure $2 \mathrm{~A}$. It is clear that there is no significant difference in the basal levels of MET25 RNA in CPF1 and cpf1 strains and that transcription of MET25 is induced in both strains when the methionine in the medium is exhausted. We conclude that CPF1 is not required for induction or regulation of MET25 transcription.

GAL2 transcription is inducible in cpf1 strains. Transcription of GAL2 is regulated by carbon source; transcription is induced by galactose and repressed by glucose in the medium (18). The CDEI site in the GAL2 promoter lies just upstream of the two GAL4 binding sites and is bound by CPF1 in vivo $(14,18)$. We had previously shown that a yeast strain such as YAG90 (cpf1) would grow well on medium containing galactose as the carbon source (14). A Northern blot was prepared from RNA isolated from DBY745 (CPF1) or YAG90 (cpf1) grown in the presence of 2\% galactose or $2 \%$ glucose (figure $2 \mathrm{~B}$ ). GAL2 mRNA is clearly inducible in the presence or absence of CPF1. However, both the repressed and derepressed levels of the GAL2 mRNA may be marginally higher in the absence of CPF1 (figures 2B). The slight increase in repressed and derepressed GAL2 mRNA levels in $c p f 1$ strains was seen in three separate experiments but its significance is unclear.

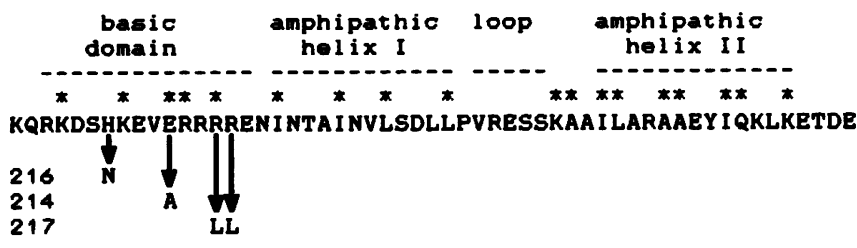

Figure 4. Point mutations created in the basic domain of CPF1. The highly conserved amino acids are indicated by asterisks.
TRP1 mRNA initiation is accurate in cpf1 strains. The three major mRNA initiation sites at the downstream TRPI promoter occur close to a CDEI motif $(40,44,45)$. Deletions or mutations in the CDEI element are sufficient to alter the usage of RNA initiation sites (figure $3 \mathrm{~A}$ and $3 \mathrm{~B}$ ). Furthermore, we have shown that changes to the chromatin structure around the RNA initiation site are seen in strains lacking cpf1 (14). We examined the pattern of RNA initiation site usage from both the chromosomal copy of TRPI (figure 3C) and the TRPI promoter and deleted derivatives on an expression vector pMA1557 (figure 3B; 40). We were unable to detect any differences in the usage of RNA initiation sites in the downstream TRPI promoter in the presence or absence of CPF1 (figure $3 \mathrm{~B}$ and $3 \mathrm{C}$ ).

\section{Construction and characterisation of DNA binding mutants} Creation of DNA binding mutants by site-directed mutagenesis. We have created a number of mutations in the CPF1 protein and analysed the phenotypes associated with them both in vivo and in vitro. Three molecules, created by site-directed mutagenesis of highly conserved residues in the basic region of CPF1 are discussed here (figure 4). Molecule 216 has the histidine at amino acid 227 mutated to asparagine (CAT to AAT). Histidine 227 is a conserved residue in the basic region of all the HLH proteins known to bind to the core motif CACRTG including upstream stimulatory factor USF (21), PHO4 (46), TFE-B (47), TFE-3 (48) and C-myc (25). The change to asparagine was made as
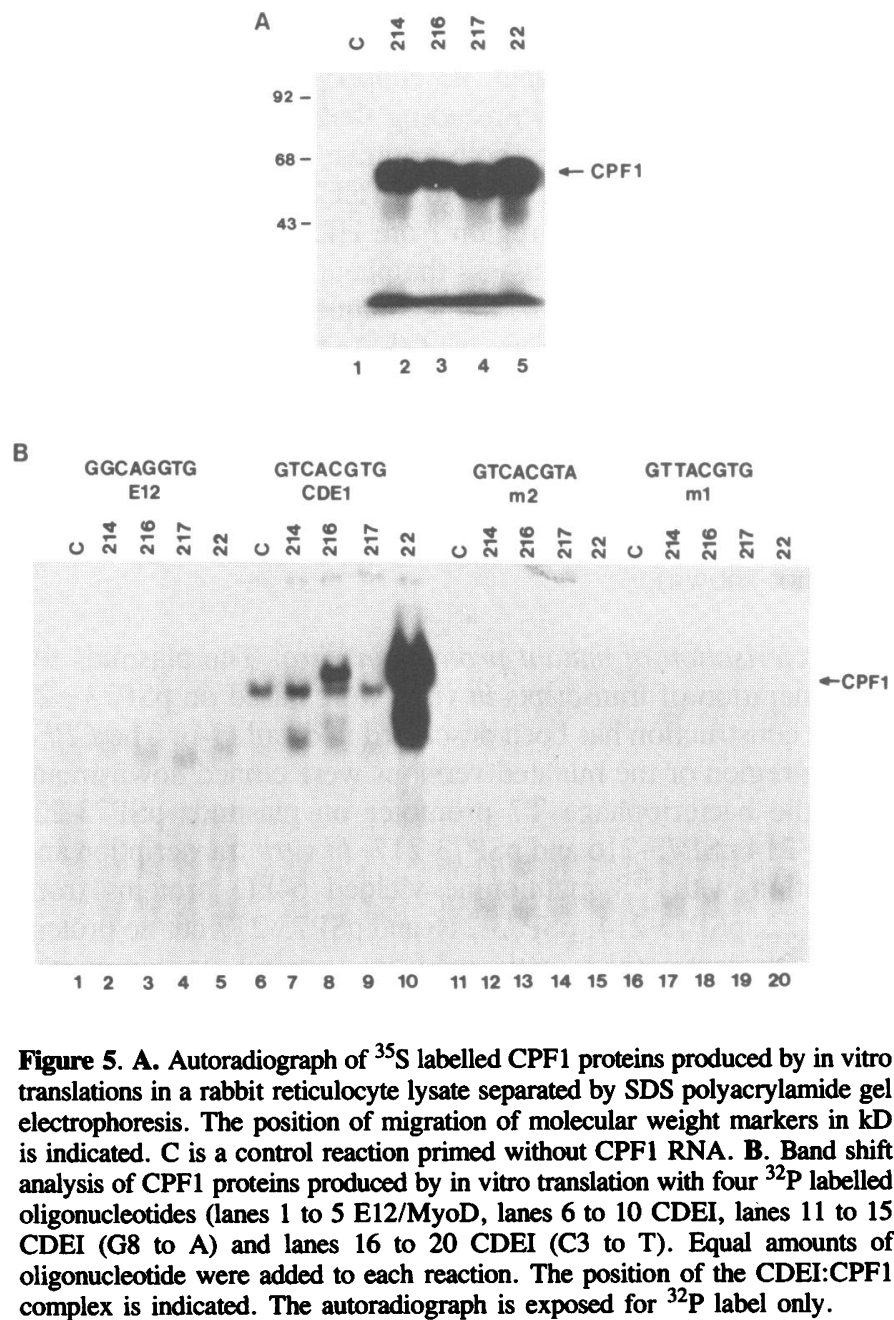


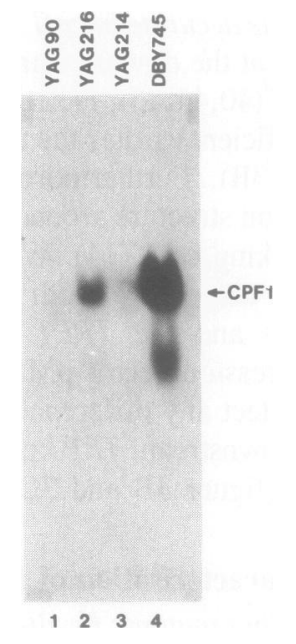

Figure 6. Band shift analysis of nuclear protein extracts prepared from strains YAG90 (lane 1), YAG216 (lane 2), YAG214 (lane 3) and DBY745 (lane 4) and incubated with ${ }^{32} \mathrm{P}$ labelled CDEI oligonucleotide. The amount of protein in each extract was monitored by the formation of a DNA:protein complex using an oligonucleotide containing a RAP1 binding site (not shown). The nuclear extract from DBY745 was diluted ten fold prior to incubation with the CDE oligonucleotide. The position of migration of the CPF1:CDEI complex is indicated.

a number of HLH proteins, including the constitutive factor E12, known to bind to the core motif CAGGTG, contain this amino acid at an equivalent position (20;23). Molecule 214 has the glutamic acid at amino acid 231 mutated to alanine (GAA to GCA). This glutamic acid is conserved in every protein containing a basic region preceding the HLH isolated to date $(23 ; 49)$. Molecule 217 has both arginine 234 and arginine 235 mutated to leucine (CGCCGC to CTCCTC). Arginine 234 is conserved in every basic region from HLH proteins isolated to date $(23,49)$. In order to assess the phenotypes associated with mutations in CPF1 in vivo, the technique of gene replacement together with $5^{\prime}$-fluoro-orotic acid (FOA) selection was used to target the mutated versions of the $C P F 1$ gene into the $C P F 1$ locus marked with the $U R A 3$ gene $(19,29)$. The presence of single base changes at the CPF1 locus was confirmed by oligonucleotide hybridization to restricted total genomic DNA (33) and, where appropriate, by the loss of restriction sites within the $C P F 1$ gene (data not shown).

Characterisation of mutant proteins in vitro. The plasmids for the generation of transcripts in vitro were based on pSP73-22 whose construction has been described in detail (14). The CPF1 coding region or the mutated versions were cloned downstream from the bacteriophage $\mathrm{T} 7$ promoter on plasmids pSP73-22, pSP73-214 pSP73-216 and pSP73-217. In vitro transcription and translation with ${ }^{35} \mathrm{~S}$ methionine yielded $64 \mathrm{kD}$ proteins from pSP73-22, pSP73-214, pSP73-216 and pSP73-217 but no protein at an equivalent position on the gel from a control reaction primed without CPF1 specific RNA (figure 5A).

Specificity of DNA binding. An in vitro gel retardation assay was used to examine the specificity of DNA:protein interactions between CDEI sequences and mutated versions of the CPF1 protein (14). In addition, a number of mutated CDEI sequences were tested for their ability to form DNA:protein interactions with all four versions of the CPF1 protein in a band shift assay. This was done to see if the specificity of the protein:DNA
Table II. Doubling times of strains containing DNA binding mutations in CPF1

\begin{tabular}{|c|c|c|c|}
\hline $\begin{array}{l}\text { Strain } \\
\text { (a) }\end{array}$ & $\begin{array}{l}\text { Nos of } \\
\text { cultures (b) }\end{array}$ & $\begin{array}{l}\text { Doubling } \\
\text { by } \mathrm{OD} 600\end{array}$ & $\begin{array}{l}\text { in minutes (c) } \\
\text { by viable cell count }\end{array}$ \\
\hline YAG214 & 8 & $138+/-6$ & $115+/-2$ \\
\hline YAG216 & 8 & $130+/-6$ & $115+1-2$ \\
\hline DBY745 & 8 & $140+/-6$ & $107+1-5$ \\
\hline YAG90 & 7 & $220+/-12$ & $153+/-5$ \\
\hline
\end{tabular}

(a) Strains were transformed with CEN based plasmid pRS315.

(b) The doubling times were measured using the same cultures as those for estimating plasmid loss rates as shown in Table III.

(c) Doubling times were determined from semilog plots.

interaction was changed. An oligonucleotide that shows specific DNA binding with the E12 homodimer or an E12/myoD heterodimer (AGCAGGTG) was included (figure 5B; 23). Three CDEI sequences were tested, 100 and $80 \mathrm{bp} C D E I$ fragments from CEN3 (GTCACATG; 50) and CEN6 (ATCACGTG) respectively and a 23 bp CDE1 oligonucleotide (GTCACGTG), and all showed the expected retardation patterns with the wild type CPF1 protein (figure 5B and data not shown). No retardation of the ${ }^{32} \mathrm{P}$ labelled CDEI DNAs by mutant proteins 214 or 217 could be seen even on very long exposures. However, mutant protein 216 ( $H$ to $N$ ) produced a faint retardation signal with all three CDEI DNA sequences that was about twenty fold less abundant than the wild type signal (figures 5B). The band seen in tracks 6 to 10 is a protein, possibly USF (21), present in the reticulocyte lysates that binds to the CDEI oligonucleotide. However, the specific DNA:CPF1 protein complex migrates more slowly. No significant binding of any of the four CPF1 proteins to CDEI sequences mutated at positions 3 (C to $T$ ) and 8 (G to $A$ ) was detected, although wild type CPF1 binds with low affinity (50 fold less than wild type) to the CDEI oligonucleotide mutated at position 8 ( $G$ to $A$ ) (figure $5 B$ ). No specific retardation of the myoD/E12 oligonucleotide was seen with any of the CPF1 proteins (figure 5B). This result indicates that all three changes in the basic region of CPF1 have significant effects on the ability of CPF1 to bind to CDEI DNA sequences. Using a limited range of CDEI-like sequences, we were also unable to detect any marked changes in the specificity of DNA binding amongst the mutant proteins.

DNA binding mutants are phenotypically methionine prototrophs and show normal growth rate

Two strains of yeast, YAG214 and YAG216, containing single point mutations at the $C P F 1$ locus were constructed. Nuclear extracts were prepared from cells grown in rich medium and tested in a gel retardation assay using ${ }^{32} \mathrm{P}$ labelled CDEI oligonucleotide. The results paralleled those from the in vitro binding assay; extracts from YAG 214 produced no detectable retardation product while extracts from YAG 216 contained a very weak binding activity towards the CDEI oligonucleotide (figure 6). The strains were tested for their auxotrophic requirements. Significantly, in contrast to the cpfl strain YAG90, neither YAG214 or YAG216 showed any requirement for methionine (see Table III). The doubling time of the cultures was measured by optical density at $600 \mathrm{~nm}$ and by viable cell counts (Table II). YAG214 and YAG216 showed growth rates that did not differ significantly from that of the parental CPF1 strain DBY745 while YAG90, the $c p f 1$ strain, grew more slowly by about $30 \%$ (13). The difference in the growth rate and the possibility of a slower cell division time rather than a loss in 


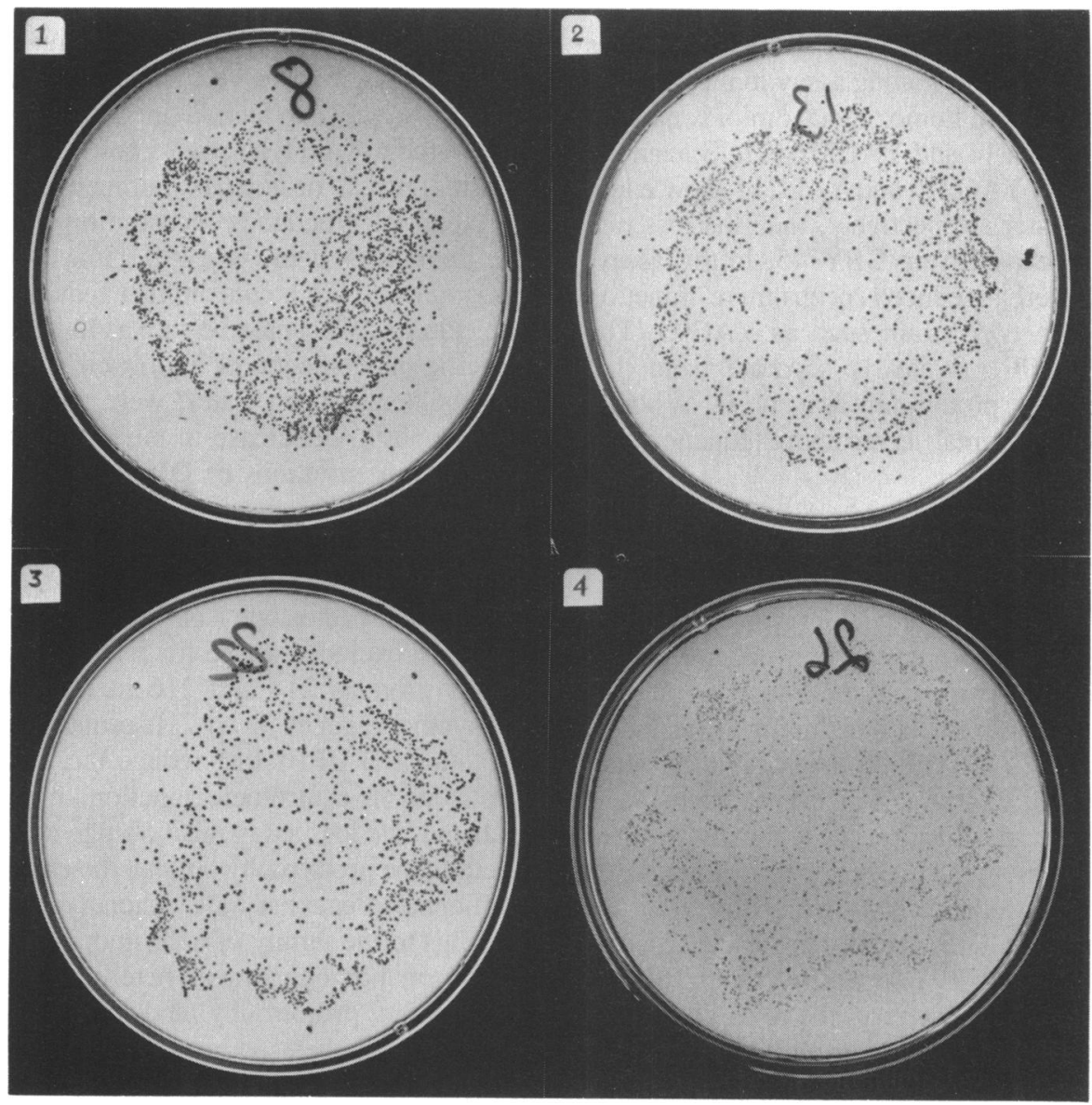

Figure 7. Strains YAG214 (1), YAG216 (2), DBY745 (3) and YAG90 (4) grown on YEPD plates for 36 hours at $30^{\circ} \mathrm{C}$. The cells are from the same cultures $(t=0)$ used to estimate the rate of loss of minichromosomes from these strains.

Table III. Phenotypes of strains containing DNA binding mutations in CPF1.

\begin{tabular}{lllllll}
\hline Strain (a) & $\begin{array}{l}\text { Binding } \\
\text { to CDEI (b) }\end{array}$ & $\begin{array}{l}\text { Methionine } \\
\text { phenotype }\end{array}$ & $\begin{array}{l}\text { Growth } \\
\text { rate }\end{array}$ & \multicolumn{2}{c}{$\begin{array}{l}\text { Loss of CEN plasmid in non-selective medium } \\
\text { No. of gen. }\end{array}$} \\
& & & & $\begin{array}{l}\text { \% cells with } \\
\text { plasmid(c) }\end{array}$ & Loss rate(d) \\
\hline YAG214 & - & Met + & +++ & 10.15 & $58.72(8)$ & 0.0511 \\
YAG216 & + & Met + & +++ & 10.15 & $81.65(8)$ & 0.0198 \\
DBY745 & +++ & Met + & +++ & 10.05 & $88.21(8)$ & 0.0124 \\
YAG90 & - & Met - & + & 8.43 & $28.16(7)$ & 0.139 \\
\hline
\end{tabular}

(a) Strains were transformed with the CEN based plasmid pRS315 (6Kb).

(b) Determined by band-shift assay as described in figure 5 .

(c) Several independent transformants were assayed, the numbers of which are given in brackets.

(d) Average plasmid loss rate per cell and per generation.

Details of the mitotic plasmid stability assays are given in Materials and methods.

viability is exemplified by the small colony size of YAG90 compared to YAG214, YAG216 and DBY745 (Figure 7).

\section{DNA binding mutants show different rates of minichromosome loss}

Rates of minichromosome loss were determined for the wild type strain DBY745, the $c p f 1$ strain YAG90 and the two strains containing CPF1 DNA binding mutants as described previously but using the 6.0Kb LEU2, CEN6, ARSH4 based plasmid pRS315 (Table III; 14,27,41). Surprisingly, the rate of loss of plasmid pRS315 from strain YAG216 shows only a marginal increase over that seen for the wild type strain DBY745, while the rate of loss of the plasmid from the cpfl strain YAG90 is about ten fold greater than from DBY745, similar to our previous results with these strains (14). In the strain YAG214, the loss rates were intermediate between DBY745 and YAG90, just over four times that seen in the wild type strain. Taken together with the DNA binding data, this would suggest that there is a correlation between DNA binding and the maintenance of centromere function. Strain YAG214 in which no DNA binding activity is detectable shows an increased rate of minichromosome loss when compared to strain YAG216 in which DNA binding is just detectable in vitro. 
The impairment in centromere function in strains containing point mutations at amino acids 227 ( $\mathrm{H}$ to $\mathrm{N}$ ) and 231 ( $\mathrm{E}$ to $\mathrm{A}$ ) has been confirmed using a quantitative mating assay to measure the rate of loss of chromosome III in homozygous diploids derived from DBY745, YAG90, YAG214 and YAG216 (12,13; data not shown). Strains derived from YAG216 and YAG214 show a loss rate 3 fold and 10 fold greater, respectively, than the loss rate from the wild type strain derived from DBY745. In this assay, the CPF1 mutant 214 has a reduced centromere function equivalent to that seen in a cpfl strain such as YAG90. The centromere function for CPF1 is clearly separate from that required to maintain methionine prototrophy as a non-DNA bound form of CPF1 shows suboptimal centromere function but methionine prototrophy.

\section{DISCUSSION}

Yeast strains lacking the centromere and promoter factor 1 (CPF1) are methionine auxotrophs. Strong circumstantial evidence that CPF1 might be involved in the transcription of at least one of the genes involved in methionine/S-adenosyl methionine biosynthesis, MET25, comes from two sources. First, the CDEI elements in the MET25 promoter had been shown to be associated with the upstream activation site and to be required for the induction of transcription in the absence of methionine/Sadenosyl methionine (16). Second, marked changes in the chromatin structure around the CDEI elements in the MET25 gene could be seen in strains lacking CPF1 suggesting that CPF1 did interact at these sites in vivo (14). However, the results presented here show clearly that the methionine auxotrophy in cpfl strains cannot be accounted for by non-induction of the MET25 gene. CPF1 is very unlikely to be acting as a transcriptional activator at an upstream activation site on the MET25 promoter. Thus, any role for CPF1 in transcriptional activation of methionine/Sadenosyl methionine biosynthetic genes remains obscure. We also examined the transcription of two other genes where CPF1 was known to be bound in vivo; GAL2 and TRP1 (14). In contrast to MET25, the CDEI sites in these promoters were outside the transcription activation sites; in TRPI the CDEI site lay close to mRNA initiation site and in GAL2 upstream from the known UAS. In both cases, as with MET25, we were unable to show any significant effect on the levels, accuracy or induction of transcription. Although this limited survey does not rule out a heterodimeric form of CPF1 binding to a CDEI site and activating transcription at some other site it strongly suggests that DNA bound CPF1 does not function to activate or regulate transcription at CDEI sites in these genes. Support for this comes from an analysis of mutations in the basic domain of CPF1. Non-DNA bound forms of CPF1, such as that in the strain YAG214, are able to maintain methionine prototrophy. This is further evidence that CPF1 is unlikely to be involved in transcriptional activation of methionine/S-adenosyl methionine biosynthetic genes by binding to CDEI motifs in promoters.

The methionine auxotrophy in cpfl strains is clearly a complex phenomenon and may be related directly or indirectly to growth rate. In all our mutant strains that were methionine prototrophs, including those not presented here, we saw normal growth rates. As yet we have been unable to separate these two phenotypes although our sample, including mutations in other regions of the protein, is at present quite small. Further indirect evidence comes from examining the growth curves of $c p f l$ strains supplemented with different concentrations of methionine. The growth rate of cpfl strains seems to be directly related to the methionine concentration; the lower the concentration the slower the growth rate. Although we have no direct evidence in support of this, the 30 to $35 \%$ reduction in the growth rate of cpfl strains compared to wild type strains could be caused by a defect in the sensing, uptake or utilization of methionine or sulphur containing metabolites. When the concentration of methionine in the growth medium drops below about $0.05 \mathrm{mM}$ this defect becomes more pronounced with a concomitant reduction in the growth rate of the culture. We cannot be sure as to whether this is a methionine specific phenomenon or applicable to other sulphur containing metabolites that, on plates, were able to satisfy the methionine auxotrophy. A decrease in the growth rate on plates was seen at low concentrations of DL-homocysteine, L-cysteine and Sadenosyl-L-methionine.

A form of CPF1 that does not bind to DNA, as measured by CDEI:DNA binding in vitro, is clearly capable of maintaining methionine prototrophy or normal growth rate. However, we have not excluded a role for a DNA bound form of the mutant CPF1 proteins 214 and 216 in the expression of methionine biosynthetic genes in vivo. It remains a formal possibility that mutations in CPF1 that reduce the DNA binding and cause a reduction in centromere function, measured using a sensitive plasmid rate loss assay, also change transcription relevant to the methionine prototrophy but that the change in transcription is not sufficient to cause a $\mathrm{Met}^{-}$phenotype. Alternatively, as CPF1 belongs to the family of helix-loop-helix (HLH) proteins which can form homodimers or heterodimers it is possible that the methionine prototrophy and normal growth rate might be mediated by a heterodimeric form of CPF1. The second half of this heterodimer might be formed by another as yet unidentified HLH protein. Any DNA binding specificity of the heterodimer is likely to be confined to the unknown protein although there is no reason to suppose that either the growth or methionine phenotypes are controlled in the nucleus by DNA:protein interactions.

In contrast, a DNA-bound form of CPF1 is clearly associated with a kinetochore function as monitored by the centromere plasmid rate loss assay. However, we were surprised that the CPF1 protein produced in vitro from pSP73-216 and from strain YAG 216 bound so poorly to a CDEI containing oligonucleotide and to a fragment containing CDEI from a yeast centromere. As yet we have been unable to demonstrate any change in the specificity of DNA binding for protein 216 . It is clear that there is no binding to a myoD/E12 oligonucleotide (core CAGGTG) or to oligonucleotides containing mutations at positions 3 and 8 of the CDEI consensus. However, a systematic survey of interactions between protein 216 and DNA containing point mutations in CDEI has yet to be done using band shift assays. Furthermore, it is not clear how binding in band shift assays is related to what happens in the cell. We have preliminary results which suggest that the mutant CPF1 protein in YAG216 can discriminate centromere and promoter DNA binding sites in vivo. The results we see with protein 216 and the strain YAG216 are reminiscent of a CDEI point mutation analysed by Baker et al. $(9,11)$. A point mutation converting $C 5$ to $T$ in the CDEI consensus (RTCATRTG) results in a 35 fold reduction in specific DNA binding of purified CPF1. However, no significant difference could be detected in vivo between the stability of a plasmid containing a wild type centromere or a centromere containing this point mutation in CDEI (9). Furthermore only very subtle changes in the in vivo footprint at the centromere 
containing this $\mathrm{C} 5$ to $\mathrm{T}$ point mutation compared to the wild type centromere were seen indicating a protein was bound to CDEI (11). The point mutation in the CPF1 protein 216 behaves in a similar way to the CDEI point mutation of Baker et al. and both results suggest strongly that centromere function in vivo can be maintained normally with a suboptimal CDEI:CPF1 interaction, as measured by band shift assays in vitro. The second point mutation present in strain YAG214 is associated with an increased plasmid instability when compared to YAG216 and undetectable CDEI binding in vitro. However, in this strain the centromere based plasmid is still more stable than in the cpfl disruption strain YAG90. One explanation for this might be that the mutant CPF1 protein 214 has weak residual CDEI binding in vivo that contributes weakly to centromere function but is undetectable in vitro. Alternatively, a reason to explain the difference in plasmid rate loss in YAG214 and YAG90 is the accumulation of lethal events in the slow growing YAG90 strain which leads to an overestimate of the number of plasmid loss events when compared with YAG214 which grows normally. It is interesting that both YAG214 and YAG90 show similar rates for chromosome III loss, which is a lethal event, in the quantitative mating assay.

This work has allowed us to separate the phenotypes associated with the CPF1 protein in yeast. We propose that the protein binds to the CDEI element of yeast centromeres where it functions to maintain optimal chromosome segregation. The CPF1 protein also functions in a non-CDEI:DNA bound form, possibly as a heterodimer, to maintain methionine prototrophy and normal growth rate. We are currently examining the possibility that more HLH proteins exist in yeast in the hope of unravelling the cause of the methionine prototrophy.

\section{ACKNOWLEDGMENTS}

We would like to thank Peter Philippsen and Hans Hegemann for their comments and support, Christine Barnes for help with the site directed mutagenesis of CPF1, Yolande Surdin-Kerjan for the MET25 probe, Colin Goding for the E12/myoD oligonucleotide and Ken Johnson for photography. J.M is a Wellcome Trust Senior Research Fellow in Basic Biomedical Science. S.D. acknowledges the support of an SERC studentship. This work was supported by grants from the Wellcome Trust, the SERC (GR/E8586) and Deutsche Forschungsgemeinschaft, Sonderforschungsbereich 272 .

\section{REFERENCES}

1. Heiter, P., Pridmore, R.D., Hegemann, J.H., Thomas, M., Davis, R.W. and Philippsen, P. (1985) Cell, 42, 913-921.

2. Hegemann, J.H., Shero, J.H., Cottarel, G., Philippsen, P. and Hieter, P. (1988) Mol. Cell. Biol., 8, 2523-2535.

3. Ng, R. and Carbon, J. (1987) Mol. Cell. Biol., 7, 4522-4534.

4. Carbon, J. and Clarke, L. (1984) J. Cell Sci. Suppl. 1, 43-58.

5. Cumberledge, S. and Carbon, J. (1987) Genetics, 117, 203-212.

6. Panzeri, L., Landonio, L., Stotz, A. and Philippsen, P. (1985) EMBO J., 4, $1867-1874$.

7. Cai, M. and Davis, R.W. (1989) Mol. Cell. Biol. 9, 2544-2550.

8. Hegemann, J.H., Shero, J.H., Cottarel, G., Philippsen, P, and Hieter, P. (1986) Mol. Gen. Genet. 205, 305-311.

9. Baker, R.E., Fitzgerald-Hayes, M. and O'Brien, T.C. (1989) J. Biol. Chem., $26410843-10850$.

10. Jiang, W. and Philippsen, P. (1989) Mol. Cell. Biol., 9, 5585-5593.

11. Densmore, L., Payne, W.E. and Fitzgerald-Hayes, M. (1991) Mol. Cell. Biol. 11, 154-165.
12. Cai, M. and Davis, R.W. (1990) Cell, 61, 437-446.

13. Baker, R.E. and Masison, D.C. (1990) Mol. Cell. Biol., 10, 2458-2467.

14. Mellor, J., Jiang, W., Funk, M., Rathjen, J., Barnes, C.A., Hinz, T., Hegemann, J.H. and Philippsen, P. (1990) EMBO J., 9, 4017-4026.

15. Bram, R.J. and Kornberg, R.D. (1987) Mol. Cell. Biol. 7, 403-409.

16. Thomas, D., Cherest, H. and Surdin-Kerjan, Y. (1989) Mol. Cell. Biol., 9, 3292-3298.

17. Kim, S., Mellor, J., Kingsman, A.J. and Kingsman, S.M. (1988) Mol. Gen. Genet., 211, 472-476,

18. Bram, R.J., Lue, N.F. and Kornberg, R.D. (1986) EMBO J., 5, 603-608.

19. Rothstein, R.J. (1983) Methods Enzymol., 101, 202-211

20. Murre, C., McCaw, P, S. and Baltimore, D. (1989) Cell, 56, 777-783.

21. Gregor, P.D., Sawadogo, M. and Reoder, R.G. (1990) Genes Dev., 4, $1730-1740$.

22. Hu, Y-F., Luscher, B., Admon, A., Mermod, N. and Tjian, R. (1990) Genes Dev., 4, 1741-1752.

23. Murre, C., McCaw, P.S., Vaessin, H., Caudy, M., Jan, L.Y., Jan, Y.N., Cabrerar, C.V., Buskin, J.N., Hauschka, S.D., Lasser, A.B., Weintraub, H. and Baltimore, D. (1989) Cell, 58, 537-544.

24. Davis, R.L., Cheng, P-F., Lassar, A.B. and Weintraub, H. (1990) Cell, 60, 733-746.

25. Prendergast, G.C. and Ziff, E.B. (1991) Science, 251, 186-189.

26. Hinnen, A., Hicks, J.B. and Fink, G.R. (1978) Proc. Natl. Acad. Sci. USA, 74, 1929-1933.

27. Sikorski, R.S. and Hieter, P. (1989) Genetics, 122, 19-27.

28. Sherman, F., Fink, G.R. and Hicks, J.B. (1979). Methods in yeast genetics; a laboratory manual. Cold Spring Habor Laboratory, Cold Spring Harbor, N.Y.

29. Boeke, J., Lacroute, F. and Fink, G. (1984) Mol. Gen. Genet., 197, $345-346$.

30. Ito, H., Fukuda, Y., Murata, K. and Kinura, A. (1983) J. Bacteriol., 153, $163-168$.

31. Holm, C., Meeks-Wagner, D.W., Fangman, W.L. and Botstein, D. (1986) Gene, 42, 169-173.

32. Southern, E.M. (1975) J. Mol. Biol., 98, 503-517.

33. Miyada, C.G. and Wallace, R.B. (1987) Methods Enzymol., 154, 94-107.

34. Dobson, M.J., Mellor, J., Fulton, A.M., Roberts, N.A., Bowen, B.A., Kingsman, S.M. and Kingsman, A.J. (1984) EMBO J., 3, 1115-1119.

35. Thomas, P. (1980) Proc. Natl. Acad. Sci. USA, 77, 5201-5205.

36. Ng, R. and Abelson, J. (1980) Proc. Natl. Acad. Sci. USA 77, 3912-3916.

37. Petes, T.D., Hereford, L.M. and Konstantin, K.G. (1978) J. Bacteriol., 134, 295-305.

38. Tschopp, J.F., Emr, S.D., Field, C. and Schekman, R. (1986) J. Bacteriol. 166, 313-318.

39. Rathjen, J. and Mellor, J. (1990) Nucl. Acids Res., 18, 3219-3225.

40. Kim, S., Mellor, J., Kingsman, A.J. and Kingsman, S.M. (1986) Mol. Cell. Biol., 6, 4251-4258.

41. Koshland, D., Kent, J.C. and Hartwell, L.H. (1985). Cell, 40, 393-403.

42. Jones, E.W. and Fink, G.R. (1982) In Strathern, J.N., Jones, E.W. and Broach, J.R. (ed.), The molecular biology of the yeast Saccharomyces: metabolism and gene expression, Cold Spring Harbor Laboratory, Cold Spring Harbor, N.Y. pp 181-299.

43. Sangsoda, S., Cherest, H. and Surdin-Kerjan, Y. (1985) Mol. Gen. Genet., 200, 407-414.

44. Dobson, M.J., Tuite, M.F., Mellor, J., Roberts, N.A., King, R.M., Bowen, B.A., Kingsman, S.M. and Kingsman, A.J. (1983) Nucl. Acids Res., 11, 2287-2302.

45. Braus, G., Paravicini, G. and Hutter, R. (1988) Mol. Gen. Genet., 212, 495-504.

46. Vogel, K., Horz, W. and Hinnen, A. (1989) Mol. Cell. Biol., 9, 2050-2057.

47. Carr, S.C. and Sharp, P.A. (1990) Mol. Cell. Biol. 10, 4384-4388.

48. Beckmann, H., Su, L-K. and Kadesch, T. (1990) Genes Dev., 4, 167-179.

49. Luscher, B. and Eisenman, R.N. (1990) Genes Dev., 4, 2025-2035.

50. Fitzgerald-Hayes, Clark, L. and Carbon, J. Cell, 29, 235-244. 